ISSN 0103-5150

Fisioter. Mov., Curitiba, v. 26, n. 3, p. 525-536, jul./set. 2013

Licenciado sob uma Licença Creative Commons

\title{
Análise de parâmetros funcionais pulmonares e da qualidade de vida na revascularização do miocárdio
}

\author{
Analysis of pulmonary functional parameters and health-related \\ quality of life in patients submitted to coronary arterial bypass graft
}

\section{Raquel Annoni ${ }^{[a]}$, Wilton Rodrigues Silva ${ }^{[b]}$, Mariana de Souza Mariano ${ }^{[c]}$}

[a] Doutora em Ciências pela Faculdade de Medicina da Universidade de São Paulo (FM/USP), docente do curso de Fisioterapia da Universidade do Vale do Sapucaí (Univas), Pouso Alegre, MG - Brasil, e-mail: rqannoni@yahoo.com.br

[b] Acadêmico do curso de Fisioterapia da Universidade do Vale do Sapucaí (Univas), Pouso Alegre, MG - Brasil, e-mail: wilton.fisioterapia@hotmail.com

[c] Acadêmica do curso de Fisioterapia da Universidade do Vale do Sapucaí (Univas), Pouso Alegre, MG - Brasil, e-mail: mariana.s.mariano@hotmail.com

\section{Resumo}

Introdução: A cirurgia de revascularização do miocárdio resulta em importantes alterações na força e função dos músculos respiratórios e na qualidade de vida de indivíduos submetidos a tal procedimento. Objetivos: Comparar a força muscular respiratória, o pico de fluxo expiratório e a qualidade de vida no pré e no pós-operatório de pacientes submetidos à revascularização do miocárdio e analisar a correlação destes parâmetros com a mecânica pulmonar e a capacidade funcional no período pós-operatório. Materiais e métodos: Avaliou-se a força muscular respiratória, o pico de fluxo expiratório e a qualidade de vida de 12 pacientes submetidos a revascularização do miocárdio no pré-operatório e no quinto dia pós-operatório. 0 teste de caminhada de 6 minutos e avaliação da mecânica pulmonar foram analisados apenas no pós-operatório. Resultados: Houve aumento da pressão expiratória máxima, do pico de fluxo expiratório e da qualidade de vida no pós-operatório em relação ao pré-operatório, sem diferenças na pressão inspiratória máxima. 0 pico de fluxo expiratório correlacionou-se positivamente com a pressão expiratória máxima. A qualidade de vida pré-operatória associou-se com o gênero e com a resistência das vias aéreas. Não houve correlação entre os fatores analisados no pré-operatório com a complacência pulmonar e com o teste de caminhada de 6 minutos. Conclusão: Pacientes submetidos a cirurgia de revascularização do miocárdio apresentam aumento da força muscular expiratória, do pico de fluxo expiratório e da qualidade de 
vida em comparação com o período anterior à cirurgia. Tais parâmetros não são bons preditores de complacência pulmonar e de capacidade funcional no período pós-operatório.

Palavras-chave: Revascularização miocárdica. Músculos respiratórios. Qualidade de vida. Mecânica respiratória.

\begin{abstract}
Introduction: The coronary arterial bypass graft (CABG) leads to important respiratory muscle function and quality of life alterations of patients undergoing this surgery. Objectives: To compare respiratory muscle strength, peak expiratory flow rate and quality of life in patients undergoing CABG and analyze the correlation between these parameters and pulmonary mechanics and functional capacity in the postoperative period. Materials and methods: We assessed respiratory muscle strength, peak expiratory flow and quality of life of twelve patients submitted to CABG preoperatively and on the fifth postoperative day. The six-minute walk test and assessment of lung mechanics were performed only postoperatively. Results: There were increases in maximal expiratory pressure, peak expiratory flow rate and quality of life postoperatively compared to preoperative values. No differences were observed in maximal inspiratory pressure. There was a positive correlation between peak expiratory flow rate and maximal expiratory pressure. The quality of life preoperatively was related with the gender and the airway resistance. There was no correlation between the factors analyzed preoperatively with the pulmonary compliance and to six-minute walk test. Conclusion: Patients undergoing coronary artery bypass graft have increased expiratory muscle strength, peak expiratory flow rate and quality of life compared with the period prior to surgery. These parameters are not good predictors of pulmonary compliance and functional capacity.
\end{abstract}

Keywords: Myocardial revascularization. Respiratory muscles. Quality of life. Respiratory mechanics.

\section{Introdução}

A cirurgia de revascularização do miocárdio é o procedimento cirúrgico mais comumente realizado no coração (1), com aproximadamente 21 mil procedimentos realizados no Brasil em 2010, computando gastos que ultrapassaram os 200 milhões de reais e taxa de mortalidade de 5,4\% (2). Embora, nos últimos anos, as técnicas cirúrgicas tenham evoluído, principalmente devido à realização da cirurgia sem circulação extracorpórea, que parece atenuar a resposta inflamatória sistêmica e as complicações advindas desse processo, as complicações pulmonares pós-operatórias permanecem constantes (3) e o impacto psicoemocional relevante (4).

As alterações funcionais pulmonares após a cirurgia de revascularização do miocárdio têm sido extensivamente exploradas e muitos estudos mostram que há diminuição da força muscular inspiratória e expiratória no pós-operatório imediato que parece não retornar ao normal até a alta hospitalar (5-7). Os volumes pulmonares também sofrem alterações importantes decorrentes da cirurgia cardíaca. Acredita-se que tais alterações se devem ao prolongado tempo em posição supina intraoperatória combinada aos efeitos anestésicos. Acrescenta-se que esses efeitos parecem favorecer o deslocamento cranial do diafragma, o relaxamento da parede torácica e a transposição do volume sanguíneo do abdome para o tórax que, juntos, levam a alterações na mecânica pulmonar e, consequentemente, a reduções na capacidade vital e na capacidade residual funcional desses pacientes (8).

A medida de pico de fluxo expiratório (PFE) é um método simples, não invasivo, econômico e rápido para avaliar a força e a velocidade de saída do ar l/ min. (9). Acredita-se que o pico de fluxo expiratório tenha relação direta com a força muscular respiratória e com o volume pulmonar. Portanto, uma vez que inúmeros autores descrevem redução significativa do volume expiratório forçado no primeiro segundo $\left(V F_{1}\right)$ e da capacidade vital forçada (CVF) em pacientes submetidos a revascularização do miocárdio (10, 11), espera-se que o PFE também esteja diminuído nessa população. Entretanto, a hipótese de relação entre essa medida espirométrica e força muscular respiratória tem sido pouco estudada. 
0 teste de caminhada de seis minutos avalia a capacidade funcional do paciente. Existe uma correlação linear entre a distância total percorrida e o consumo de oxigênio de pico (12). Esse teste, de fácil realização e de baixo custo, tem sido amplamente utilizado como preditor de melhora em casos de intervenções medicamentosas e/ou cirúrgicas em pacientes pneumopatas e cardiopatas.

Apesar de oferecer riscos e complicações cirúrgicas, a revascularização do miocárdio tem a potencialidade de melhorar a qualidade de vida dos pacientes. A Organização Mundial de Saúde define qualidade de vida como "um estado de completo desenvolvimento físico, mental e bem-estar social e não meramente a ausência de doença" (13). Alguns estudos têm associado ansiedade e depressão pré-operatória à maior morbidade e pior qualidade de vida após a cirurgia cardíaca $(14,15)$.

Muitos estudos têm abordado os parâmetros funcionais pulmonares, a capacidade funcional e qualidade de vida de pacientes submetidos a revascularização do miocárdio separadamente, entretanto, nenhum trabalho, até então, interligou esses dados e os correlacionou a fim de mostrar se algum desses parâmetros influencia ou sofre influência de outro. Portanto, os objetivos deste estudo foram comparar a força muscular respiratória, o pico de fluxo expiratório e a qualidade de vida no pré e no pós-operatório de pacientes submetidos a revascularização do miocárdio e analisar a correlação desses parâmetros com a mecânica pulmonar e a capacidade funcional no período pós-operatório.

\section{Materiais e métodos}

O projeto de pesquisa foi aprovado pelo Comitê de Ética em Pesquisa da Universidade do Vale do Sapucaí (n. 1477/10 e n. 1531/11). Todos os participantes receberam informações sobre os procedimentos realizados no estudo e assinaram um Termo de Consentimento Livre e Esclarecido, respeitando todas as normas e diretrizes da Resolução 196/96 do Conselho Nacional de Saúde (CNS).

\section{Amostra}

Foram estudados 20 pacientes, brasileiros, de ambos os sexos, submetidos à cirurgia de revascularização do miocárdio e internados na enfermaria de cardiologia do Hospital das Clínicas Samuel Libânio (HCSL), Pouso Alegre, (MG). Destes, foram excluídos por se recusarem a participar da avaliação pós-operatória. Não foram incluídos pacientes que realizaram outro procedimento cirúrgico juntamente com a cirurgia de revascularização do miocárdio, com história de doença neuromuscular, neurológica ou psiquiátrica.

\section{Avaliação da força muscular respiratória}

A força muscular respiratória foi avaliada no período pré-operatório e no quinto dia pós-operatório, por meio da mensuração das pressões inspiratórias (PImax) e expiratórias máximas (PEmáx). Para tanto, utilizou-se um manovacuômetro analógico (Gerar/ Brasil) e foram realizadas três manobras de mensuração, sendo anotada a de melhor valor. Foram utilizados os valores de referência descritos por Costa et al. (16).

Para a realização da técnica de manovacuometria, os pacientes foram posicionados adequadamente sentados, com o tronco num ângulo de $90^{\circ} \mathrm{com}$ as coxas, os pés e as costas apoiados no chão e no encosto da cadeira, respectivamente, e os membros superiores apoiados sobre os membros inferiores ou segurando a traqueia no aparelho.

Para verificação da PImáx os pacientes realizaram uma respiração normal através de um bucal, com o nariz ocluído por um oclusor nasal. Após o comando, os pacientes foram encorajados a realizar uma expiração profunda (até o volume residual) e, em seguida, efetuar um esforço inspiratório máximo contra a via aérea ocluída. Para verificação da PEmáx, os pacientes foram encorajados a realizar uma inspiração profunda (até a capacidade pulmonar total) e, em seguida, efetuar um esforço expiratório máximo contra a via aérea ocluída.

\section{Avaliação do pico de fluxo expiratório}

Para a avaliação do pico de fluxo expiratório no pré e no quinto dia pós-operatório, utilizou-se o dispositivo Peak Flow Meter Mini Wright (Clement Clarke, Harlow, Inglaterra), com uma escala de 0 a 9001/min. Os pacientes foram posicionados adequadamente sentados (mesma postura descrita para a avaliação da força muscular respiratória). Após a 
oclusão nasal o paciente foi encorajado a respirar calmamente através do bocal e, em seguida, foi solicitada uma expiração forçada máxima a partir da capacidade pulmonar total. Essa manobra foi realizada três vezes (com espaços regulares entre as medidas) e considerou-se aquela de maior valor.

\section{Avaliação da qualidade de vida}

Para avaliação da qualidade de vida, utilizou-se o instrumento genérico Medical Outcomes Study 36 item Short-Form Health Survey (SF-36), idealizado por Ware e Sherboune em 1992 (17) e adaptado para o português por Ciconelli et al. em 1997 (18). O questionário foi aplicado no pré e no quinto dia pós-operatório de maneira clara e sucinta para que os pacientes compreendessem as perguntas. 0 instrumento é composto por 36 questões que abordam oito domínios divididos em dois grandes componentes: o componente físico, que envolve a capacidade funcional (10 itens), a dor (2 itens), o estado geral de saúde (5 itens) e o aspecto físico (4 itens); e o componente mental, que abrange a saúde mental (5 itens), o aspecto emocional (3 itens), o aspecto social ( 2 itens) e a vitalidade (4 itens). Para avaliação dos resultados, foi utilizado um escore próprio de cada questão, e esses valores foram normatizados em uma escala de zero a 100. Menores valores refletem má percepção da saúde, perda da função e presença de dor, enquanto maiores valores refletem uma boa percepção da saúde, função preservada e ausência de dor.

\section{Teste de caminhada de 6 minutos}

0 teste de caminhada de 6 minutos foi realizado de acordo com padronização da American Thoracic Society $(18,19)$. 0 teste foi realizado apenas no pós-operatório e supervisionado por um avaliador treinado. Os pacientes foram encorajados a percorrer a maior distância possível em um corredor de aproximadamente 50 metros, e a caminharem em um ritmo de média intensidade verificado com base na frequência cardíaca, frequência respiratória, saturação periférica de oxigênio e pela escala de percepção de dispneia de Borg (20). Os sujeitos foram orientados a interromperem o teste caso sentissem sintomas como dispneia, tonturas, angina, sudorese intensa, taquicardia ou dores musculoesqueléticas. A distância percorrida durante o teste foi registrada em metros. Foram utilizados os valores de referência descritos por Opasich et al. (21).

Todos os testes e o questionário de qualidade de vida foram aplicados pelo mesmo avaliador, tanto no período pré-operatório quanto no pós-operatório. A ordem de realização dos testes foi escolhida de forma randômica.

\section{Avaliação da mecânica pulmonar}

A avaliação da mecânica pulmonar foi realizada no pós-operatório imediato, ou seja, até uma hora depois da entrada do paciente na Unidade de Terapia Intensiva (UTI) do HCSL, e todos os pacientes estavam monitorizados e estáveis hemodinamicamente. Foram avaliados os fatores da mecânica pulmonar - complacência pulmonar e resistência das vias aéreas. Os pacientes permaneceram em decúbito dorsal horizontal em sedação contínua em bomba de infusão, conforme prescrição médica. Todos estavam intubados e foram ventilados mecanicamente no ventilador mecânico Dixtal DX3010 ${ }^{\circledR}$ ou Servo-S ${ }^{\circledR}$, em modalidade controlada, ciclado a volume, com volume corrente (VC) de $6 \mathrm{ml} / \mathrm{kg}$, frequência respiratória (f) de $12 \mathrm{rpm}$, PEEP de $8 \mathrm{cmH}_{2} \mathrm{O}, \mathrm{FiO}_{2}$ de $60 \%$ e fluxo inspiratório de $60 \mathrm{l} / \mathrm{min}$. Pausa inspiratória de $2 \mathrm{~s}$ foi realizada a fim de verificar os valores de pressão de platô (Pplato) do sistema respiratório. Além disso, foram verificados a pressão de pico inspiratório (PPI) e o volume corrente exalado (VCex). As medidas de mecânica pulmonar foram obtidas a partir dos seguintes cálculos: complacência dinâmica Cdin = VC / PPI - PEEP; complacência estática Cest = VC / Pplato - PEEP, resistência das vias aéreas Raw = PPI - Pplato / fluxo inspiratório.

\section{Análise estatística}

A análise estatística foi realizada por meio do programa SPSS (SPSS, Chigago / EUA) versão 15.0. 0 teste de Kolmogorov-Smirnov foi utilizado para testar a normalidade da amostra. Para a comparação da força muscular respiratória e pico de fluxo expiratório no pré-operatório e no pós-operatório foi utilizado o teste $t$ pareado. Os valores do questionário de qualidade de vida antes e após a cirurgia de revascularização do miocárdio foram comparados por meio do teste de Wilcoxon. 
0 teste de correlação de Spearman foi realizado para avaliar as correlações entre força muscular respiratória, pico de fluxo expiratório, teste de caminhada de 6 minutos, mecânica pulmonar e medidas do teste de qualidade de vida. 0 nível de significância foi estabelecido como $\mathrm{p} \leq 0,05$.

\section{Resultados}

Doze pacientes submetidos a revascularização do miocárdio, com idade média de 65,4 \pm 9,5 anos, participaram do estudo. Destes, seis eram do sexo feminino; dois eram tabagistas, três ex-tabagistas e sete referiram nunca haver fumado. Em relação ao etilismo dois pacientes classificaram-se como ex-etilistas e dez relataram não ter costume de ingerir bebida alcoólica.

0 diagnóstico médico prévio à cirurgia de revascularização do miocárdio foi de insuficiência coronariana (seis pacientes), angina estável (três), angina estável e insuficiência coronariana concomitantes (dois), aterosclerose coronariana (um). As principais comorbidades dos pacientes estudados foram: hipertensão arterial sistêmica (59\%), diabetes mellitus (29\%) e dislipidemia (6\%). Dos 12 pacientes submetidos à revascularização do miocárdio, nove cirurgias (75\%) foram realizadas com circulação extracorpórea, com tempo médio de 80,7 \pm 19,9 minutos.

No Gráfico 1 é possível observar os valores de PImáx. e PEmáx. nos períodos pré e pós-operatório.
As médias da PImáx (A) no pré e pós-operatório foram de $-86,6 \pm 32,2 \mathrm{cmH}_{2} \mathrm{O}(158,4 \pm 84,8 \%$ predito) e $-84,16 \pm 23,9 \mathrm{cmH}_{2} \mathrm{O}(152,3 \pm 68,3 \%$ predito $)$, respectivamente $(p=0,57)$. A média da PEmáx $(B)$ no pré-operatório foi de $66,5 \pm 24,0 \mathrm{cmH}_{2} \mathrm{O}(75,6 \pm$ $23,4 \%$ predito), com aumento significativo para 76,0 $\pm 21,0 \mathrm{cmH}_{2} \mathrm{O}(87,6 \pm 24,4 \%$ predito $)$ no quinto dia pós-operatório $(\mathrm{p}=0,007)$.

0 valor médio do pico de fluxo expiratório no pré-operatório foi de $198 \pm 78 \mathrm{l} / \mathrm{min}$. com aumento significativo no período pós-operatório $211 \pm 76 \mathrm{l} /$ $\min (\mathrm{p}=0,02)$ (Gráfico 2).

As médias dos escores obtidos nos domínios do teste de qualidade de vida SF-36 no período pré-operatório variaram de 41 a 64, enquanto no período pós-operatório variaram de 61 a 70 (Gráfico 3).

Quando comparamos os escores no período pré e pós-operatório, observamos importantes modificações tanto no componente físico quanto no componente mental. No domínio da capacidade funcional, a média elevou-se de 47,2 $\pm 12,8$ no pré-operatório para $62,2 \pm 13,5$ no pós-operatório ( $p=0,02$ ). Avaliados individualmente, nenhum dos pacientes demonstrou queda na capacidade funcional pós-operatória quando comparada ao período pré-operatório.

No aspecto físico, houve aumento significativo no escore de 41,7 $\pm 12,3$ no pré-operatório para $64,6 \pm$ 12,9 no pós-operatório $(\mathrm{p}=0,01)$. Em relação à dor, a média foi de $55 \pm 4,4$ no pré-operatório e de $68,6 \pm$

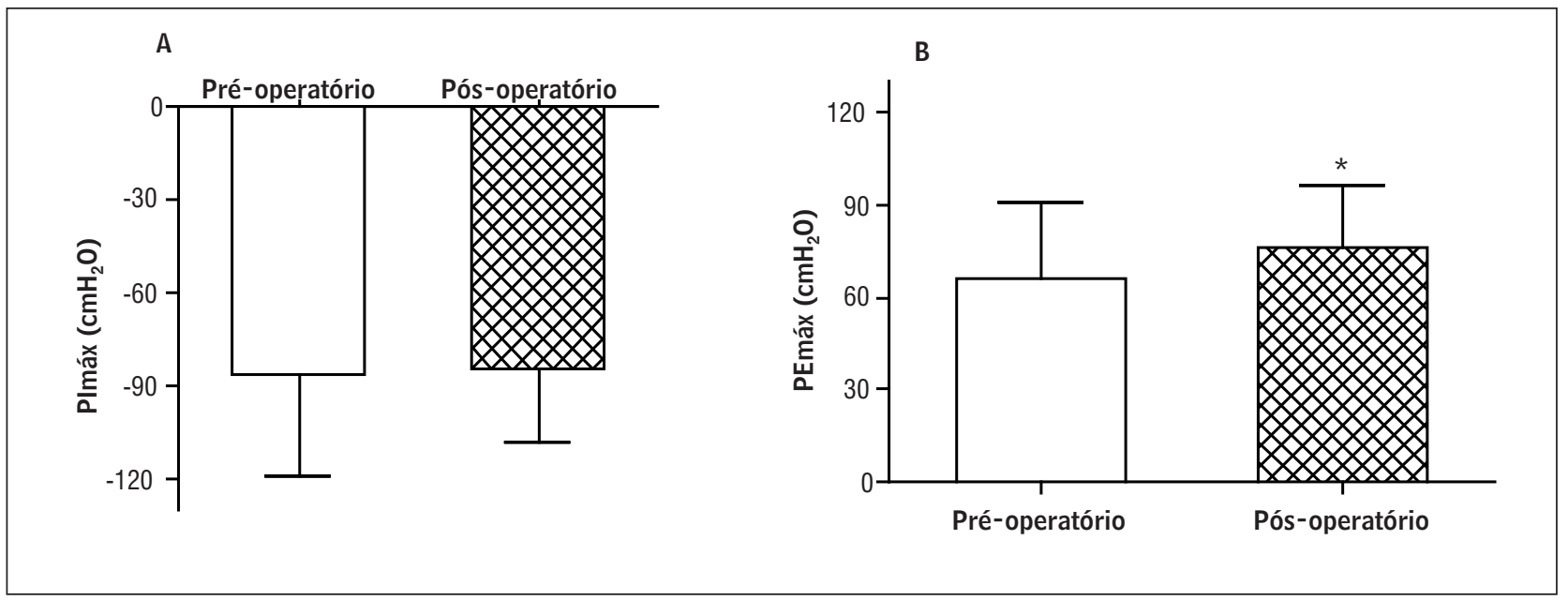

Gráfico 1- Comparação da pressão inspiratória máxima (PImáx) $[\mathrm{A}]$ e da pressão expiratória máxima (PEmáx) [B] entre 0 pré-operatório e o quinto dia pós-operatório

Fonte: Dados da pesquisa.

Nota: * $p=0,007$ em relação ao pré-operatório. 


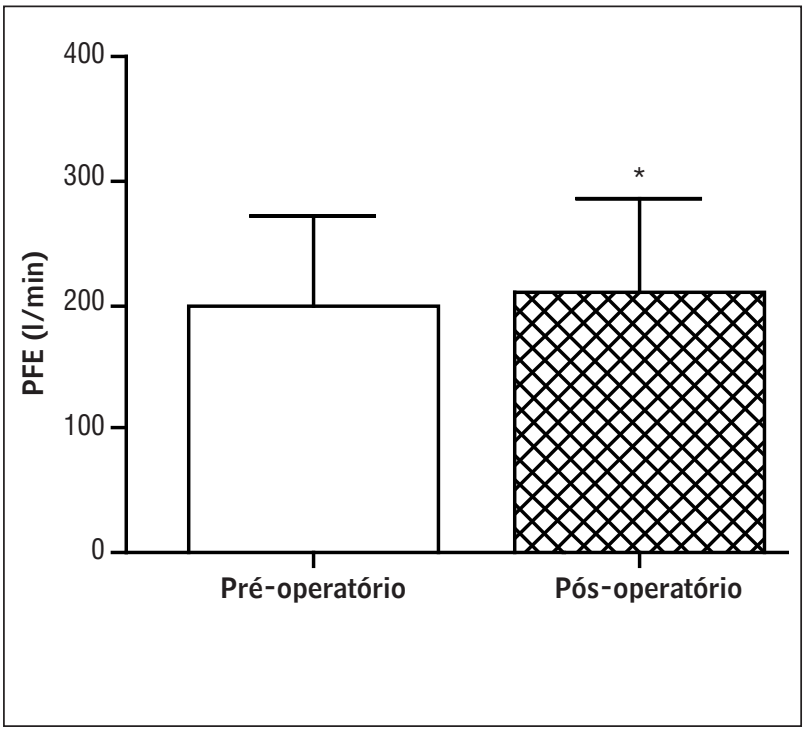

Gráfico 2 - Comparação do pico de fluxo expiratório (PFE) entre o pré-operatório e o quinto dia pós-operatório

Fonte: Dados da pesquisa.

Nota: ${ }^{*} p=0,02$ em relação ao pré-operatório.

6,7 no pós-operatório, aumento este estatisticamente significante $(p=0,02)$.

Na avaliação do estado geral de saúde, 11 pacientes (91\%) apresentaram melhora, e apenas um paciente $(9 \%)$ manteve a mesma condição. Houve aumento significativo entre a média obtida no pré-operatório $(50,3 \pm 13)$ e a pós-operatória $(62,6 \pm$ $15,4)$ com $\mathrm{p}=0,04$.

A avaliação do estado vital ou vitalidade revelou melhora significativa em todos os pacientes, passando de 49,2 $\pm 5,1$ no pré-operatório para $61,7 \pm 9,1$ no pós-operatório $(\mathrm{p}=0,04)$.

Embora o escore de aspecto social tenha aumentado de $64,6 \pm 12,9$ no pré-operatório para 70,3 \pm 9,7 no pós-operatório, não se apurou diferença estatística nesse domínio $(p=0,08)$. As modificações do aspecto emocional revelaram melhora em seis pacientes (50\%) e manutenção do quadro em outros seis. Mesmo assim, constatou-se diferença entre as médias obtidas no pré e no pós-operatório $(50 \pm 17,8$ e 67, respectivamente) $\operatorname{com} p=0,01$. Por fim, em relação à saúde mental, observou-se melhora significativa entre o período pré e o pós-operatório $(\mathrm{p}=0,02)$, com aumento do escore de $50 \pm 16,3$ para $69,3 \pm 13$, 8 .

As variáveis de mecânica respiratória e troca gasosa (complacência estática e dinâmica, resistência das vias aéreas, relação $\mathrm{PaO}_{2} / \mathrm{FiO}_{2}$ e $\mathrm{PaCO}_{2}$ ) bem como o volume corrente exalado e o resultado do teste de

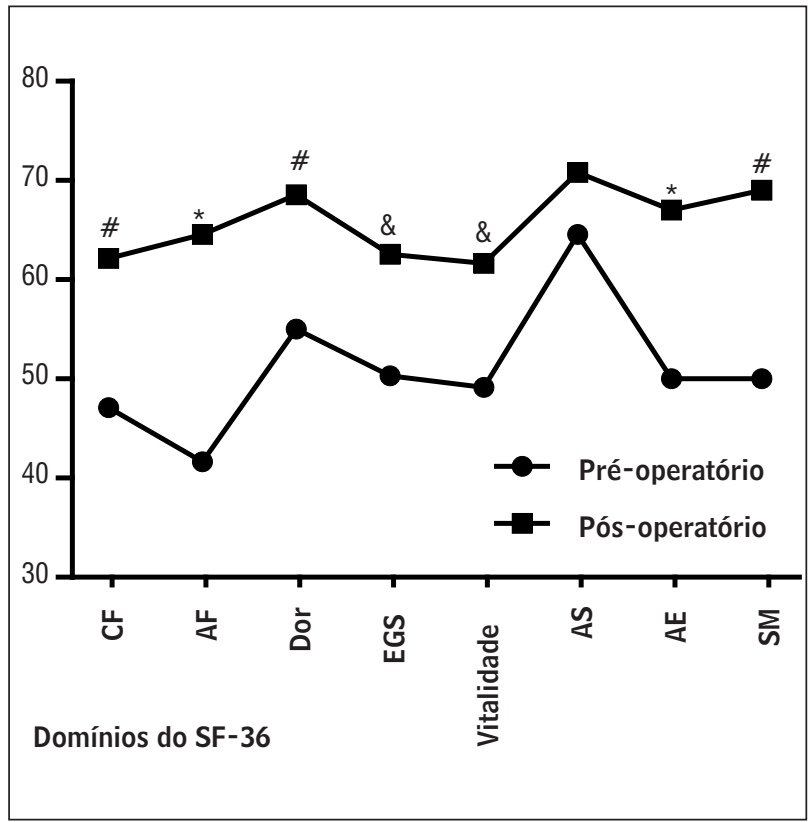

Gráfico 3 - Comparação dos domínios do SF-36 entre o pré-operatório e o quinto dia pós-operatório

Legenda: $\mathrm{CF}=$ capacidade funcional; $\mathrm{AF}=$ aspectos físicos; $\mathrm{EGS}$ = estado geral de saúde; $A S=$ aspectos sociais; $A E=$ aspectos emocionais; $\mathrm{SM}=$ saúde mental; ${ }^{\star}=\mathrm{p}<$ 0,01 em relação ao pré-operatório; \# $=p=0,02$ em relação ao pré-operatório; $\&=p=0,04$ em relação ao pré-operatório.

caminhada de 6 minutos estão demonstrados na Tabela 1. Em relação à normalidade $(21,22)$, observou-se que as médias dos valores de complacência estática e dinâmica estavam abaixo do normal, enquanto que a resistência das vias aéreas encontrava-se dentro dos limites de normalidade. Os índices de troca gasosa $\left(\mathrm{PaO}_{2} / \mathrm{FiO}_{2}\right.$ e $\left.\mathrm{PaCO}_{2}\right)$ apresentavam valores considerados normais.

\section{Correlações clínicas}

A pressão expiratória máxima no pré-operatório correlacionou-se positivamente com o pico de fluxo expiratório pré-operatório e pós-operatório $(R=0,70$ e $p=0,01 ; R=0,72$ e $p=0,009$, respectivamente). Da mesma forma, a PEmáx no pós-operatório mostrou correlação com o pico de fluxo expiratório mensurada no pré-operatório e no pós-operatório $(\mathrm{R}=0,82$ e $\mathrm{p}=$ 0,$001 ; R=0,81$ e $p=0,002$, respectivamente).

Houve correlação positiva entre gênero e o estado geral de saúde avaliada no pré-operatório $(R=0,61 \mathrm{ep}=0,034)$, sendo que homens apresentaram 
Tabela 1 - Parâmetros de mecânica pulmonar, oxigenação e da distância percorrida no teste de caminhada de 6 minutos no pós-operatório

\begin{tabular}{lcc}
\hline & Valores obtidos & Valores de referência (22) \\
\hline Cest $\left(\mathrm{ml}^{\prime} / \mathrm{cmH}_{2} \mathrm{O}\right)$ & $55 \pm 12$ & $60-100$ \\
$\mathrm{Cdin}\left(\mathrm{ml} / \mathrm{cmH}_{2} \mathrm{O}\right)$ & $40 \pm 4$ & $50-80$ \\
$\mathrm{Raw}\left(\mathrm{cmH}_{2} \mathrm{O} / / \mathrm{s}\right)$ & $3 \pm 1$ & $2-5$ \\
$\mathrm{PaO}_{2} / \mathrm{FiO}_{2}$ & 256 & $>200$ \\
$\mathrm{PaCO}_{2}(\mathrm{mmHg})$ & 45 & $35-45$ \\
$\mathrm{VCex}(\mathrm{ml})$ & $547 \pm 73$ & - \\
$\mathrm{TC6M}($ metros$)$ & $108 \pm 63,3$ & - \\
\hline
\end{tabular}

Legenda: Cest $=$ média dos valores de complacência estática; $C$ din $=$ média dos valores de complacência dinâmica; Raw = resistência das vias aéreas; $\mathrm{PaO}_{2} / \mathrm{FiO}_{2}=$ relação; $\mathrm{PaCO}_{2}=$ relação; $\mathrm{VCex}=$ volume corrente exalado no período pós-operatório; $\mathrm{TC} 6 \mathrm{M}=$ volume do teste de caminhada de 6 minutos no período pós-operatório.

Fonte: Opasich et al. (21).

média de $58,6 \pm 13,3$ e mulheres $42 \pm 5,5$ nesse quesito. A resistência das vias aéreas mostrou correlação negativa com os aspectos sociais pré-operatórios $(\mathrm{R}=-0,70$ e $\mathrm{p}=0,012)$.

Não houve correlações clínicas estatisticamente significantes entre a mecânica pulmonar (complacência estática, complacência dinâmica e resistência das vias aéreas) com os outros parâmetros analisados (idade, PImáx, PEmáx, pico de fluxo expiratório e teste de caminhada de 6 minutos).

\section{Discussão}

O presente estudo demonstrou aumento da força muscular expiratória e do pico de fluxo expiratório no quinto dia pós-operatório, bem como a correlação entre essas duas variáveis, em pacientes submetidos a revascularização do miocárdio. Além disso, melhora na qualidade de vida foi observada em todos os aspectos estudados, exceto no aspecto social. 0 domínio aspecto social (pré-operatório) correlacionou-se com a resistência das vias aéreas. Houve uma correlação positiva entre o gênero e o fator estado geral de saúde avaliado no período pré-operatório.

Embora muitos estudos demonstrem perda de força muscular respiratória após cirurgias cardíacas $(5,6)$, sabe-se que a mensuração da força muscular respiratória por manovacuometria tem como limitação a necessidade de colaboração do avaliado. Silva et al. (5) observaram maior perda de força muscular no primeiro dia pós-operatório com aumento progressivo até a alta hospitalar, porém sem recuperação total em relação ao achado pré-operatório. Acredita-se que parte da diminuição de força muscular esteja relacionada às alterações estruturais da caixa torácica secundárias à incisão operatória, tais como perda de parte da pressão negativa intrapleural (devido ao acúmulo de líquidos e a abertura da caixa torácica) e diminuição da complacência pulmonar (pelo edema alveolar e atelectasias), culminando em biomecânica desfavorável à geração de força pelos músculos respiratórios (7). Nosso estudo demonstrou aumento da pressão expiratória máxima no quinto dia pós-operatório, o que pode ser explicado pelo fator aprendizado e colaboração do paciente.

Barros et al. (6) estudaram dois grupos de pacientes submetidos a cirurgia cardíaca e verificaram que o grupo que recebeu treinamento muscular respiratório no pós-operatório apresentou maior pressão expiratória máxima na alta hospitalar comparado ao período pré-operatório, diferentemente do grupo controle. Nossos pacientes não receberam treinamento muscular respiratório específico no pós-operatório; entretanto, em nosso serviço, todos os pacientes submetidos a cirurgia cardíaca são acompanhados pela equipe de fisioterapia que prescreve exercícios terapêuticos individualizados ao menos duas vezes ao dia, o que pode ter influenciado nos resultados. 
O pico de fluxo expiratório representa o fluxo máximo de ar que pode ser gerado durante uma expiração forçada, iniciado a partir da capacidade pulmonar total (23). A medida do PFE parece ter uma boa relação com o $\operatorname{VEF}_{1}(10,11)$ e com pico de fluxo da tosse em indivíduos sem doença obstrutiva ou restritiva (24). A efetividade da tosse, ou seja, a capacidade de remoção de secreções brônquicas e/ ou corpos estranhos das vias aéreas depende da magnitude do fluxo expiratório; entretanto, é comum a avaliação da força de tosse ser realizada apenas por meio da mensuração da força muscular expiratória (25). Embora esta seja uma medida indireta, alguns estudos observaram que em indivíduos com pressões expiratórias máximas baixas, o pico de fluxo expiratório também apresentava valores abaixo da normalidade $(5,6)$. Nosso estudo corrobora e complementa esses estudos, uma vez que demonstramos uma correlação positiva entre o pico de fluxo expiratório com a pressão expiratória máxima. Entretanto, nos estudos citados, tanto a PE máxima quanto o pico de fluxo expiratório diminuíram após a intervenção cirúrgica, diferentemente de nossos resultados; isso sugere que a força dos músculos expiratórios é essencial para a geração do fluxo expiratório. Freitas et al. (26) estudaram idosos ativos e moderadamente ativos e verificaram que o pico de fluxo expiratório, o pico de fluxo da tosse, a PI máxima e a PE máxima foram maiores nos ativos em relação aos moderadamente ativos. Uma vez que não foi avaliado o estado funcional dos pacientes participantes deste estudo, não se pode afirmar que houve relação da atividade física com o aumento do pico de fluxo expiratório.

0 enfraquecimento do diafragma decorrente de sua manipulação durante as cirurgias cardíacas é uma complicação bem reconhecida na literatura. As razões desse dano são múltiplas: lesão e/ou inibição reflexa do nervo frênico, lesão nas fibras musculares pela incisão cirúrgica, mudança na relação tensão/ comprimento dos músculos respiratórios, anestesia e dor (7). Inúmeros estudos mostraram um declínio na capacidade dos músculos inspiratórios de gerarem força em comparação dos valores pré-operatórios $(5,6,27)$. Clergue et al. (28) observaram contração dos músculos abdominais à expiração em pacientes submetidos a cirurgias cardíacas em associação a um aumento da atividade dos músculos intercostais externos sem aumento na ativação do diafragma, o que demonstra uma clara alteração no equilíbrio muscular respiratório normal. 0 presente estudo corrobora os estudos citados anteriormente, pois também observamos diminuição da força muscular inspiratória em nossos pacientes, porém sem diferenças estatísticas. Entretanto, acreditamos que tal redução tenha pouca significância clínica, uma vez que, ao calcularmos o valor predito da PI máxima, de acordo com Costa et al. (16), verificamos que nossos pacientes apresentavam valores preditos acima de $100 \%$ tanto no período pré-operatório quanto no pós-operatório.

A avaliação de qualidade de vida relacionada à saúde reflete a maneira como os indivíduos percebem e reagem ao seu estado de saúde e aos outros aspectos de suas vidas (29). 0 questionário SF-36, instrumento genérico para avaliação da qualidade de vida, é dividido em dois grandes componentes: físico e mental. Para o componente físico, altos escores indicam nenhuma limitação física, deficiência ou mal-estar, e refletem um alto nível de energia. Já escores baixos sugerem limitações no autocuidado, atividades físicas, no papel social, dor corporal importante ou cansaço frequente. Altos escores no componente mental apontam ausência de sofrimento psicológico ou limitações em atividades sociais e/ou emocionais, ao passo que escores baixos indicam sofrimento psicológico frequente e incapacidade social devido a problemas emocionais (29).

A avaliação da qualidade de vida por meio do questionário SF-36 no período pré-operatório revelou que os pacientes avaliados neste estudo apresentavam baixos escores em todos os componentes estudados, com maior comprometimento dos componentes capacidade funcional, aspectos físicos e vitalidade. Isso demonstra o alto impacto da doença no desempenho das atividades diárias e profissionais, principalmente devido à falta de energia e à fadiga durante atividades físicas que exigem esforço, como tomar banho e vertir-se. Gois et al. (30) demonstraram maior comprometimento nos aspectos físicos, emocionais, capacidade funcional e dor na avaliação pré-operatória de pacientes cardiopatas submetidos a cirurgia de revascularização do miocárdio.

A implicação da percepção da qualidade de vida pré-operatória nos resultados físicos e psicológicos após a cirurgia cardíaca tem motivado inúmeros estudos sobre o tema (29-31). Um estudo multicêntrico que avaliou a associação de fatores da qualidade de vida com mortalidade e duração da internação hospitalar mostrou que escores pré-operatórios baixos para o componente mental foram fortemente associados com a mortalidade e com longa permanência 
hospitalar; já escores pré-operatórios baixos para o componente físico associaram-se a um tempo prolongado de internação hospitalar (29). Depressão e ansiedade também são fatores preditivos de pior recuperação após cirurgias cardíacas $(32,33)$.

Entretanto, a melhora da qualidade de vida de pacientes coronariopatas após a cirurgia de revascularização do miocárdio mostra a efetividade do tratamento cirúrgico $(34,35)$. 0 presente estudo corrobora esses resultados, uma vez que demonstra melhora na qualidade de vida em todos os fatores estudados, exceto os aspectos sociais, no momento anterior à alta hospitalar. É possível que a melhora na qualidade de vida se deva à redução do quadro de ansiedade, medo e desconfiança presente antes do procedimento cirúrgico, modificando positivamente a expectativa do paciente em relação à sua saúde futura.

Uma forte associação entre o gênero e o domínio estado geral de saúde pré-operatório foi observada em nosso estudo. Em uma coorte que avaliou 759 pacientes que seriam submetidos à revascularização do miocárdio, os autores notaram que as mulheres tinham maior limitação do que os homens em atividades físicas básicas (como levantar da cama ou de uma cadeira) ou em atividades físicas mais complexas (como caminhada ou subida e descida de escadas). Além disso, apresentavam maior restrição a atividades sociais e mais sintomas de depressão e ansiedade do que pacientes do sexo masculino. As mulheres eram menos propensas a se casar, tinham rendimentos inferiores aos dos homens, eram mais velhas e apresentavam estado emocional menos favorável antes da cirurgia (36). Esses resultados, juntamente com os nossos achados, sugerem que as mulheres apresentam estado clínico, social e emocional pré-operatório menos favorável do que os homens; assim, medidas terapêuticas para minimizar as complicações pós-operatórias nessa população deveriam ser tomadas antes das cirurgias.

As complicações pulmonares decorrentes da cirurgia de revascularização do miocárdio parecem estar ligadas a alterações mecânicas e inflamatórias. No presente estudo, observamos diminuição da complacência estática e dinâmica e acreditamos que tais alterações possam ser atribuídas a inúmeros mecanismos. A cisão do esterno danifica a estabilidade da caixa torácica, levando à diminuição da complacência pulmonar. Além disso, a artéria mamária interna, usada como enxerto nas cirurgias de revascularização do miocárdio, parece fornecer o suprimento sanguíneo para a parede posterior do esterno e, uma vez dissecada, tal suprimento estaria comprometido. Ademais, para a utilização desse enxerto a pleura precisa ser aberta; muitos autores acreditam que a pleurotomia contribua para a alteração da mecânica pulmonar no pós-operatório de cirurgias cardíacas devido à perda de equilíbrio entre as forças elásticas do pulmão e a caixa torácica $(8,11)$.

A anestesia e o uso de circulação extracorpórea contribuem para a diminuição da complacência pulmonar e para o aumento da resistência das vias aéreas. A anestesia combinada com a posição supina prolongada resulta em deslocamento cranial do diafragma, relaxamento da parede torácica e mudança no volume de sangue do abdome para o tórax (8). Esses fatores em combinação com o uso de circulação extracorpórea, cuja inflamação aguda pulmonar e sistêmica é conhecida (37), leva ao desequilíbrio ventilação-perfusão e aumento do shunt pulmonar. Assim como em nosso estudo, Ambrozin e Cataneo (38) também demonstraram diminuição das complacências estática e dinâmica em pacientes submetidos a revascularização do miocárdio.

A resistência das vias aéreas, embora dentro dos limites de normalidade, apresentou uma relação negativa com o domínio aspectos sociais avaliado no pré-operatório. Entretanto, não houve correlação de nenhum outro parâmetro avaliado no pré-operatório com a mecânica pulmonar pós-operatória. É possível que o número de pacientes participantes deste estudo não tenha sido suficiente para os cálculos estatísticos, demonstrando baixo poder de nossa amostra. Outra explicação possível é que fatores como força muscular respiratória e pico de fluxo expiratório não sejam bons índices preditores de piora da mecânica pulmonar após a cirurgia de revascularização do miocárdio. Para esclarecer a ausência de associações entre essas variáveis sugerem-se novos estudos com maior número de indivíduos.

No anseio de procurar índices que estimem piores condições pós-operatórias em cirurgias cardíacas, inúmeros estudos fracassaram em encontrá-los. Ambrozin e Cataneo (38) separaram os pacientes submetidos a revascularização do miocardio em dois grupos conforme condições pré-operatórias. Tais condições, como antecedentes de doença pulmonar, sintomas respiratórios, comorbidades e carga tabágica, não foram estimadores de pior mecânica pulmonar no pós-operatório (38). Nozawa et al. (39) demonstraram que a mecânica respiratória e os índices de oxigenação não são bons parâmetros para predizer o sucesso do desmame da ventilação mecânica em pacientes cardiopatas. No entanto, 
os mesmos autores discorrem que disfunção cardíaca e tempo de circulação extracorpórea parecem interferir no sucesso do desmame de ventilação mecânica (39). Almeida et al. (40) observaram que choque cardiogênico, disfunção renal e o tipo de cobertura à internação mostraram-se importantes preditores de mortalidade e de intercorrência grave observadas no intraoperatório.

Nosso estudo tem algumas limitações que devem ser consideradas. 0 pequeno número de pacientes estudados pode ter influenciado negativamente nossos resultados. Além disso, os pacientes estudados não tiveram graves complicações após a cirurgia, visto que todos receberam alta hospitalar em até 8 dias. 0 estudo de Székely et al. (29) demonstrou que, quanto pior a qualidade de vida no pré-operatório, maior o número de dias de permanência no hospital ( $>14$ dias). A comparação entre um grupo de pacientes com complicações pulmonares pós-operatórias ou com um grupo de longa permanência hospitalar poderia contribuir para uma descrição mais abrangente da associação entre fatores preditivos pré-operatórios.

\section{Conclusões}

Em resumo, os pacientes submetidos a revascularização do miocárdio apresentam aumento da força muscular respiratória expiratória e, consequentemente, do pico de fluxo expiratório, além de demonstrarem melhora na qualidade de vida, mensurada por meio dos domínios do SF-36. Esses parâmetros não são preditores da complacência pulmonar e de capacidade funcional no pós-operatório de cirurgia de revascularização do miocárdio.

\section{Referências}

1. Abu-Omar Y, Taggart DP. Off-pump coronary artery bypass grafting. Lancet. 2002;360(9329):327-30.

2. Brasil. Ministério da Saúde. Sistema de Informações Hospitalares do SUS (SIH/SUS). [acesso 23 set 2011]. Disponível em: http://tabnet.datasus.gov.br/cgi/tabcgi.exe?sih/cnv/niuf.def

3. Montes FR, Maldonado JD, Paez S, Ariza F. Off-pump versus on-pump coronary artery bypass surgery and postoperative pulmonary dysfunction. J Cardiothorac Vasc Anesth. 2004;18(6):698-703.
4. Rosenfeldt F, Braun L, Spitzer O, Bradley S, Shepherd J, Bailey M, et al. Physical conditioning and mental stress reduction -a randomised trial in patients undergoing cardiac surgery. BMC Complement Altern Med. 2011 9;11:20.

5. Silva AB, Di Lorenzo VAP, Oliveira CR, Luzzi S. Comportamento da função pulmonar e da força muscular respiratória em pacientes submetidos à revascularização do miocárdio e a intervenção fisioterapêutica. RBTI. 2004;16:155-9.

6. Barros GF, Santos CS, Granado FB, Costa PT, Límaco RP, Gardenghi G. Respiratory muscle training in patients submitted to coronary arterial bypass graft. Rev Bras Cir Cardiovasc. 2010;25(4):483-90.

7. Siafakas NM, Mitrouska I, Bouros D, Georgopoulos D. Surgery and the respiratory muscles. Thorax. 1999;54:458-65.

8. Wynne R, Botti M. Postoperative pulmonary dysfunction in adults after cardiac surgery with cardiopulmonary bypass: clinical significance and implications for practice. Am J Crit Care. 2004;13(5):384-93.

9. Boaventura CM, Amuy FF, Franco JH, Sgarbi ME, Matos LB, Matos LB. Valores de referência de medidas de pico de fluxo expiratório máximo em escolares. Arq Med ABC. 2007;32(Suppl. 2):S30-S34.

10. Morsch KT, Leguisamo CP, Camargo MD, Coronel CC, Mattos W, Ortiz LD, et al. Ventilatory profile of patients undergoing CABG surgery. Rev Bras Cir Cardiovasc. 2009;24:180-7.

11. Berrizbeitia LD, Tessler S, Jacobowitz IJ, Kaplan P, Budzilowicz L, Cunningham JN. Effect of sternotomy and coronary bypass surgery on postoperative pulmonary mechanics. Comparison of internal mammary and saphenous vein bypass grafts. Chest. 1989;96(4):873-6.

12. Araújo CO, Makdisse MRP, Peres PRT, Tebexreni AS, Ramos LR, Matsushita AM, et al. Diferentes padronizações do teste da caminhada de seis minutos como método para mensuração da capacidade de exercício de idosos com e sem cardiopatia clinicamente evidente. Arq Bras Cardiol. 2006;86(3):198-205.

13. World Health Organization, Division of mental health and prevention of substance abuse. WHOQOL: measuring quality of life. 1997. 
14. Mallik S, Krumholz HM, Lin ZQ, Kasl SV, Mattera JA, Roumains SA, et al. Patients with depressive symptoms have lower health status benefits after coronary artery bypass surgery. Circulation. 2005;111(3):271-7.

15. Tully P, Baker R, Turnbull D, Winefield H, Knight J. Negative emotions and quality of life six months after cardiac surgery: the dominant role of depression not anxiety symptoms. J Behav Med. 2009;32(6):510-22.

16. Costa D, Gonçalves HA, Lima LP, Ike D, Cancelliero KM, Montebelo MI. Novos valores de referência para pressões respiratórias máximas na população brasileira. J Bras Pneumol. 2010;36(3):306-12.

17. Ware JE Junior, Sherbourne CD. The MOS 36-item short-form health survey (SF-36). I. Conceptual framework and item selection. Med Care. 1992;30(6):473-83.

18. Ciconelli RM, Ferraz MB, Santos W, Meinão IM, Quaresma MR. Tradução para a língua portuguesa e validação do questionário genérico de avaliação de qualidade de vida SF-36 (Brasil SF-36). Rev Bras Reumatol. 1999;39(3):143-50.

19. Amenicon Thoracic Society - ATS. Committee on Proficiency Standards for Clinical Pulmonary Function Laboratories. ATS statement: guidelines for the six-minute walk test. Am J Respir Crit Care Med. 2002;166(1):111-7.

20. Borg GA. Psychophysical bases of perceived exertion. Med Sci Sports Exerc. 1982;14(5):377-81.

21. Opasich C, De Feo S, Pinna GD, Furgi G, Pedretti R, Scrutinio D, et al. Distance walked in the 6-minute test soon after cardiac surgery: toward an efficient use in the individual patient. Chest. 2004;126(6):1796-801.

22. Carvalho CRR, Franca SA, Okamoto VN. III Consenso brasileiro de ventilação mecânica. J Bras Pneumol. 2007;33(Suppl. 2):S54-S70.

23. Velloso M, Vieira DSR, Martins JA, Montemezzo D. Instrumentos da avaliação respiratória. In: Vega JM, Luque A, Sarmento GJV, Moderno LFO, organizadores. Tratado de fisioterapia hospitalar: assistência integral ao paciente. São Paulo: Atheneu; 2012. p. 57-70.

24. Leiner GC, Abramowitz S, Small MJ, Stenby VB. Cough peak flow rate. Am J Med Sci. 1966;251(2):211-4.

25. Freitas FS, Parreira VF, Ibiapina CC. Aplicação clínica do pico de fluxo da tosse: uma revisão de literatura. Fisioter Mov. 2010;23(3):495-502.
26. Freitas FS, Ibiapina CC, Alvim CG, Britto RR, Parreira VF. Relationship between cough strength and functional level in elderly. Rev Bras Fisioter. 2010;14(6):470-6.

27. Stein R, Maia CP, Silveira AD, Chiappa GR, Myers J, Ribeiro JP. Inspiratory muscle strength as a determinant of functional capacity early after coronary artery bypass graft surgery. Arch Phys Med Rehabil. 2009;90(10):1685-91.

28. Clergue F, Whitelaw WA, Charles JC, Gandjbakhch I, Pansard JL, Derenne JP, et al. Inferences about respiratory muscle use after cardiac surgery from compartmental volume and pressure measurements. Anesthesiology. 1995;82(6):1318-27.

29. Székely A, Nussmeier NA, Miao Y, Huang K, Levin J, Feierfeil $\mathrm{H}$, et al. A multinational study of the influence of health-related quality of life on in-hospital outcome after coronary artery bypass graft surgery. Am Heart J. 2011;161(6):1179-85.

30. Gois CFL, Dantas RAS, Torrati FG. Qualidade de vida relacionada à saúde antes e seis meses após a revascularização do miocárdio. Rev Gaúch Enferm. 2009;30(4):700-7.

31. Contrada RJ, Boulifard DA, Hekler EB, Idler EL, Spruill TM, Labouvie EW, et al. Psychosocial factors in heart surgery: presurgical vulnerability and postsurgical recovery. Health Psychol. 2008;27(3):309-19.

32. Underwood M, Firmin RK, Jehu D. Aspects of psychological and social morbidity in patients awaiting coronary artery bypass grafting. Br Heart J. 1993;69(5):382-4.

33. Tully P, Baker R, Turnbull D, Winefield H, Knight J. Negative emotions and quality of life six months after cardiac surgery: the dominant role of depression not anxiety symptoms. J Behav Med. 2009;32(6):510-22.

34. Nogueira CRSR, Hueb W, Takiuti ME, Girardi PBMA, Nakano T, Fernandes F, et al. Qualidade de vida após revascularização cirúrgica do miocárdio com e sem circulação extracorpórea. Arq Bras Cardiol. 2008;91(4):217-22.

35. Welke KF, Stevens JP, Schults WC, Nelson EC, Beggs VL, Nugent WC. Patient characteristics can predict improvement in functional health after elective coronary artery bypass grafting. Ann Thorac Surg 2003;75(6):1849-55. 
36. Czajkowski SM, Terrin M, Lindquist R, Hoogwerf B, Dupuis G, Shumaker SA, et al. Comparison of preoperative characteristics of men and women undergoing coronary artery bypass grafting (the Post Coronary Artery Bypass Graft [CABG] Biobehavioral Study). Am J Cardiol. 1997;79(8):1017-24.

37. Massoudy P, Zahler S, Becker BF, Braun SL, Bazankay A, Meisner H. Evidence for inflammatory responses of the lungs during coronary artery bypass grafting with cardiopulmonary bypass. Chest. 2001;119(1):31-36.

38. Ambrozin ARP, Cataneo AJM. Aspectos da função pulmonar após revascularização do miocárdio relacionados com risco pré-operatório. Braz J Cardiovasc Surg. 2005;20(4):408-15.

39. Nozawa E, Kobayashi E, Matsumoto ME, Feltrim MIZ, Carmona MJC, Auler JOC Júnior. Assessment of factors that influence weaning from long-term mechanical ventilation after cardiac surgery. Arq Bras Cardiol. 2003;80(3):306-10.
40. Almeida FF, Barreto SM, Couto BRGM, Starling CEF. Fatores preditores da mortalidade hospitalar e de complicações per-operatórias graves em cirurgia de revascularização do miocárdio. Arq Bras Cardiol. 2003;80:41-50.

Recebido: 03/02/2012

Received: 02/03/2012

Aprovado: 10/09/2012

Approved: 09/10/2012 